\title{
GEOESTATÍSTICA APLICADA À ANÁLISE DA INCERTEZA ESPACIAL ASSOCIADA À PRECIPITAÇÕES INTENSAS
}

\author{
Andrea Koga-Vicente ${ }^{(\mathrm{a})}$, Armando Zaupa Remacre ${ }^{(\mathrm{b})}$, Moacir Cornetti ${ }^{(\mathrm{c})}$ \\ (a) Centro de Pesquisas Meteorológicas e Climáticas Aplicadas a Agricultura/Faculdade de Engenharia Agrícola, \\ UNICAMP, andrea.kvicente @ gmail.com \\ (b) Departamento de Geologia e Recursos Naturais/Instituto de Geociências, UNICAMP, armando@ige.unicamp.br \\ (c) Departamento de Geologia e Recursos Naturais/Instituto de Geociências, UNICAMP, moacir@ige.unicamp.br
}

\section{Eixo: CLIMATOLOGIA EM DIFERENTES EIXOS ESCALARES: MUDANÇAS E VARIABILIDADES}

\begin{abstract}
Resumo/
A análise das incertezas inerentes à espacialização de precipitações intensas é uma informação fundamental para embasar avaliações de risco e tomadas de decisão para prevenção de impactos associados. Com intuito de contribuir para o tema, neste trabalho a simulação estocástica é empregada para a análise das incertezas de um evento pluvial intenso ocorrido na Região Metropolitana de Campinas. Mapas de desvio padrão e de áreas de probabilidade de ocorrência de chuvas a partir de $80 \mathrm{~mm} / 24 \mathrm{~h}$ foram gerados a com base na simulação de 200 cenários equiprováveis, fornecendo ferramentas para a análise de áreas com maior probabilidade de chuvas intensas em condições análogas.
\end{abstract}

Palavras chave: evento extremo de precipitação, incerteza espacial, simulação estocástica, geoestatística, cenários

\section{Introdução}

As chuvas intensas deflagram impactos que resultam em elevado número de vítimas, sendo que os desastres hidrológicos (enchentes, alagamentos e movimentos de massa úmidos) e meteorológicos (tempestades), causam cerca de 135 milhões de vítimas e US\$ 71 bilhões de danos econômicos por ano no mundo (GUHA-SAPIR et al., 2011). No Brasil, cerca de 197.000 pessoas são afetadas por ano (EmDat, 2011) principalmente no verão, quando os altos volumes de precipitação deflagram movimentos de massa, alagamentos e enchentes, atingindo de forma mais contundente a população de baixa renda (KOGA-VICENTE, 2005; ARAKI, 2007; ZANELLA et al., 2009; CASTELLANO, 2010).

O conhecimento da dinâmica da distribuição da precipitação em dada localidade pode contribuir para a prevenção de impactos decorrentes das chuvas intensas. Contudo, a variabilidade espacial e temporal inerente ao sistema atmosférico dificulta a compreensão de sua dinâmica, tornando-se uma importante fonte de incerteza na análise destas ocorrências. 
Para a realização de avaliação de risco, é necessário considerar, quantificar e modelar a incerteza espacial (MORGAN e HERION, 1990; HELTON e BURMASTER, 1996), pois uma das principais aplicações da representação da incerteza associada ao dado geoespacial é oferecer ao tomador de decisão o nível de certeza para ações.

No âmbito dos estudos dos eventos atmosféricos extremos, uma demanda importante da Defesa Civil é obter informações que subsidiem ações de evacuação de áreas sujeitas a impactos em um episódio de chuvas extremas. As evacuações, para serem efetivas, devem ocorrer em um espaço de tempo que permita a remoção das pessoas antes da deflagração do episódio. Contudo, se a operação de remoção dos habitantes é realizada e o episódio não se concretiza, suscita uma diminuição da credibilidade em relação à futuras ações; se o evento realmente acontece e a evacuação não foi realizada, os danos podem significar perda de vidas. Ou seja: a decisão de evacuação de uma população frente à uma situação de risco deve balancear o custo entre um "falso positivo" (evacuação quando ela não é necessária) e um "falso negativo" (não evacuação quando for justificado) (LAVE e APT, 2006).

Desta forma, considerar a incertezas as informações geradas pelas pesquisas sobre precipitações intensas é de importância fundamental para fornecer subsídios confíáveis e embasar a tomada de decisão, tanto nos espaços urbanos quanto agrícolas.

Sob a luz destas reflexões, o objetivo do presente trabalho é contribuir para a discussão da incerteza na representação da distribuição espacial de episódios de chuvas intensas, bem como fornecer ferramentas de apoio à tomada de decisão, utilizando como exemplo o estudo de caso de um evento pluvial extremo ocorrido na Região Metropolitana de Campinas.

\section{Metodologia}

Para este trabalho, foram utilizados dados de precipitação`em 24h da Região Metropolitana de Campinas (RMC) (Fig. 1), coletados em 19 postos componentes da rede pluviométrica do Departamento de Água e Energia Elétrica (DAEE) e do Instituto Agronômico de Campinas (IAC), órgãos de alta confiabilidade quanto à qualidade das informações. Foi selecionado um episódio de precipitação intensa ocorrido em 01/12/2003. O critério para escolha foi ter sido registrado o valor igual ou superior a $100 \mathrm{~mm} / 24 \mathrm{~h}$ em pelo menos um dos postos, montante que causa impactos significativos para a sociedade e para os sistemas físicos na região (KOGA-VICENTE, 2005). 

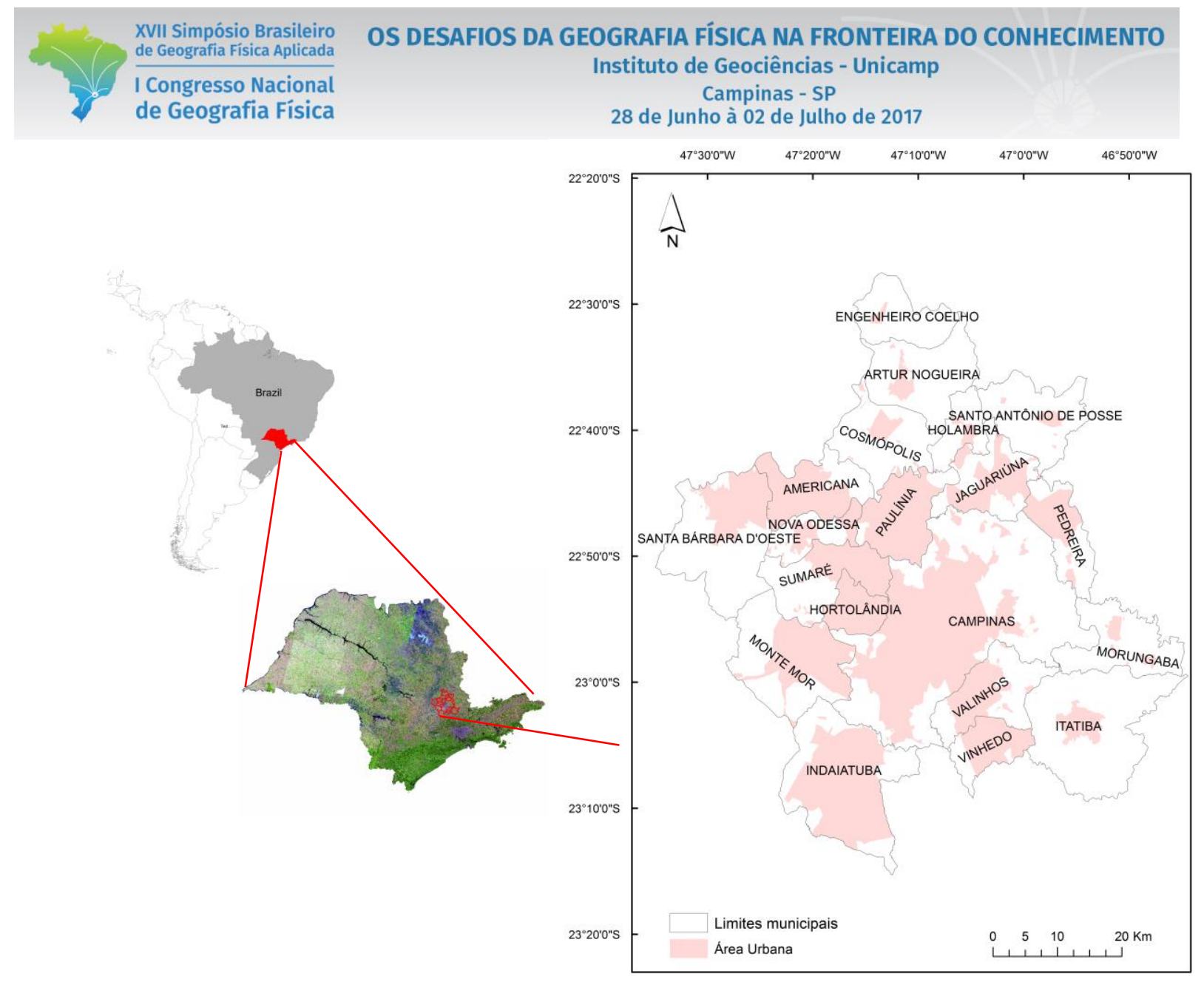

Figura 1. Municípios que compõe a RMC. Em destaque, a área urbanizada dos municípios..

Fonte dos dados: IBGE. Elaborado pelos autores

A simulação estocástica foi a técnica utilizada para quantificar as incertezas. É uma extensão espacial do conceito de simulação Monte Carlo e fornece um método para quantificar a incerteza e minimizar o risco (BABISH, 2006). É uma técnica de interpolação que gera diversas realizações ou cenários, nos quais valores de dados conhecidos são "honrados" (condicionado aos dados coletados) e cada realização é diferente das outras porque sempre há incertezas nos pontos não amostrados. Os cenários gerados são equiprováveis, ou seja, tem a mesma probabilidade de ocorrência (REMACRE e SACANI, 2005).

Os algoritmos de Simulação Sequencial Condicionada aplicam o Teorema de Bayes (JOURNEL e HUIJBREGTS, 1978), onde a variável é interpretada como realização de uma função aleatória (FA) que é obtida a partir do histograma (distribuição) e do variograma (dispersão).

As realizações da simulação foram geradas a partir da equação: 
onde,

$$
\begin{aligned}
& Z_{S}=\text { realizações } \\
& Z_{K}=\text { estimativa da krigagem simples } \\
& \sigma_{K}=\text { variância } \\
& \mathrm{U}=\text { função aleatória }
\end{aligned}
$$

Foram geradas 200 simulações e a série obtida passou por um procedimento de pós-processamento, gerando um resumo da incerteza espacial do estudo por meio de dois tipos de exibição (GOOVAERTS, 1997):

- Mapa de desvio padrão: representa os valores de desvio padrão do conjunto de realizações para cada nó da malha. Permite observar a dispersão entre as $\mathrm{N}$ realizações e reflete graus de incerteza.

- Mapas de probabilidade: mostra locais onde a variável excede determinados limiares. Para cada nó é calculado o número de realizações, nas quais o valor simulado excede ou é igual ao valor de corte estabelecido pelo pesquisador. Foi determinado o limiar de corte de $80.0 \mathrm{~mm}$ com base em estudos realizados na área (KOGA-VICENTE, 2005).

\section{Resultados e discussões}

O evento extremo de precipitação ocorrido na Região Metropolitana de Campinas no dia 01/12/2003 caracterizou-se por ter sido de grande intensidade em toda região (tabela 1). Não obstante o registro de chuvas intensas no verão, houve marcada excepcionalidade em 5 postos, com montantes acima de $100 \mathrm{~mm} / 24 \mathrm{~h}$.

O limiar a partir do qual ocorrem impactos na região foi estabelecido em 50mm/24h em estudo realizado por Koga-Vicente (2005). Em 12 dos 19 postos selecionados o volume de chuva registrado foi acima deste limiar. 
Tabela 1. Precipitação registrada nos postos da RMC em 01/12/2003



A análise do variograma mostra que a correlação espacial cessa por volta de $10 \mathrm{~km}$ (Fig. 2). Os parâmetros do modelo foram estabelecidos em alcance de 15000 e patamar de 0.96, sem efeito pepita. Esse fato chama a atenção, em virtude do caráter discreto da precipitação, contudo, explica-se por ter sido registrado baixos volumes em apenas 2 postos.

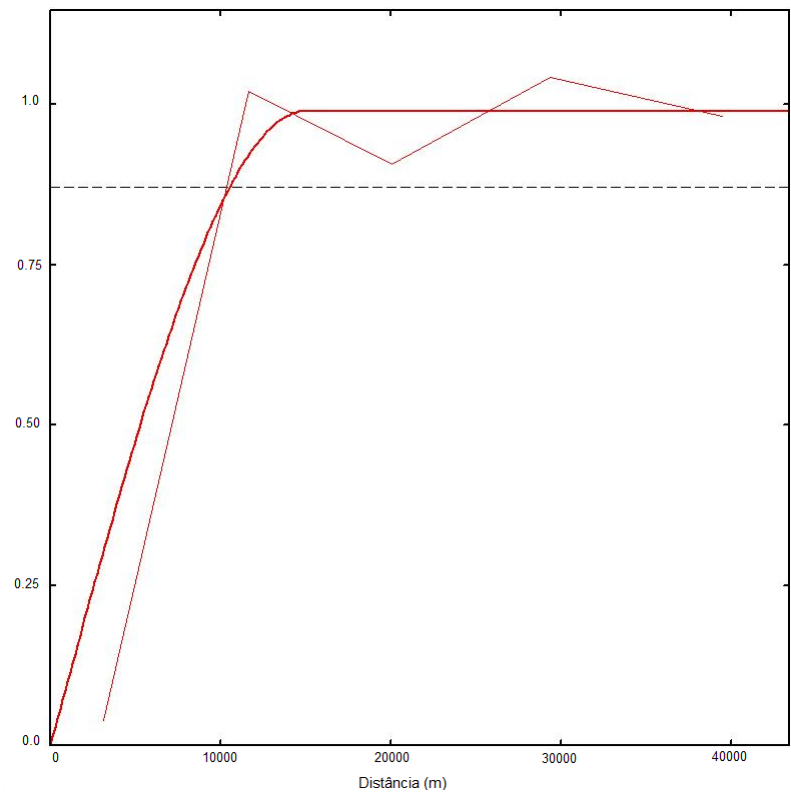

O mapa de desvio padrão (Figura 2) é um recurso importante para avaliar as áreas com maior ou menor incerteza na espacialização da precipitação e auxiliar na interpretação do mapa com a representação da variável de interesse. É possível observar que as áreas com maior certeza para interpretação são aquelas 
XVII Simpósio Brasileiro de Geografia Fisica Aplicada

I Congresso Nacional de Geografia Física
OS DESAFIOS DA GEOGRAFIA FÍSICA NA FRONTEIRA DO CONHECIMENTO

Instituto de Geociências - Unicamp

Campinas - SP

28 de Junho à 02 de Julho de 2017

com desvio padrão $=0$ (azul claro) e correspondem à localização dos postos pluviométricos, ou seja, ao ponto em que o dado foi efetivamente coletado. A medida em que o ponto se distancia do local da coleta, a incerteza aumenta pois é estimado um valor para cada nó da grade com base no histograma da distribuição dos dados.

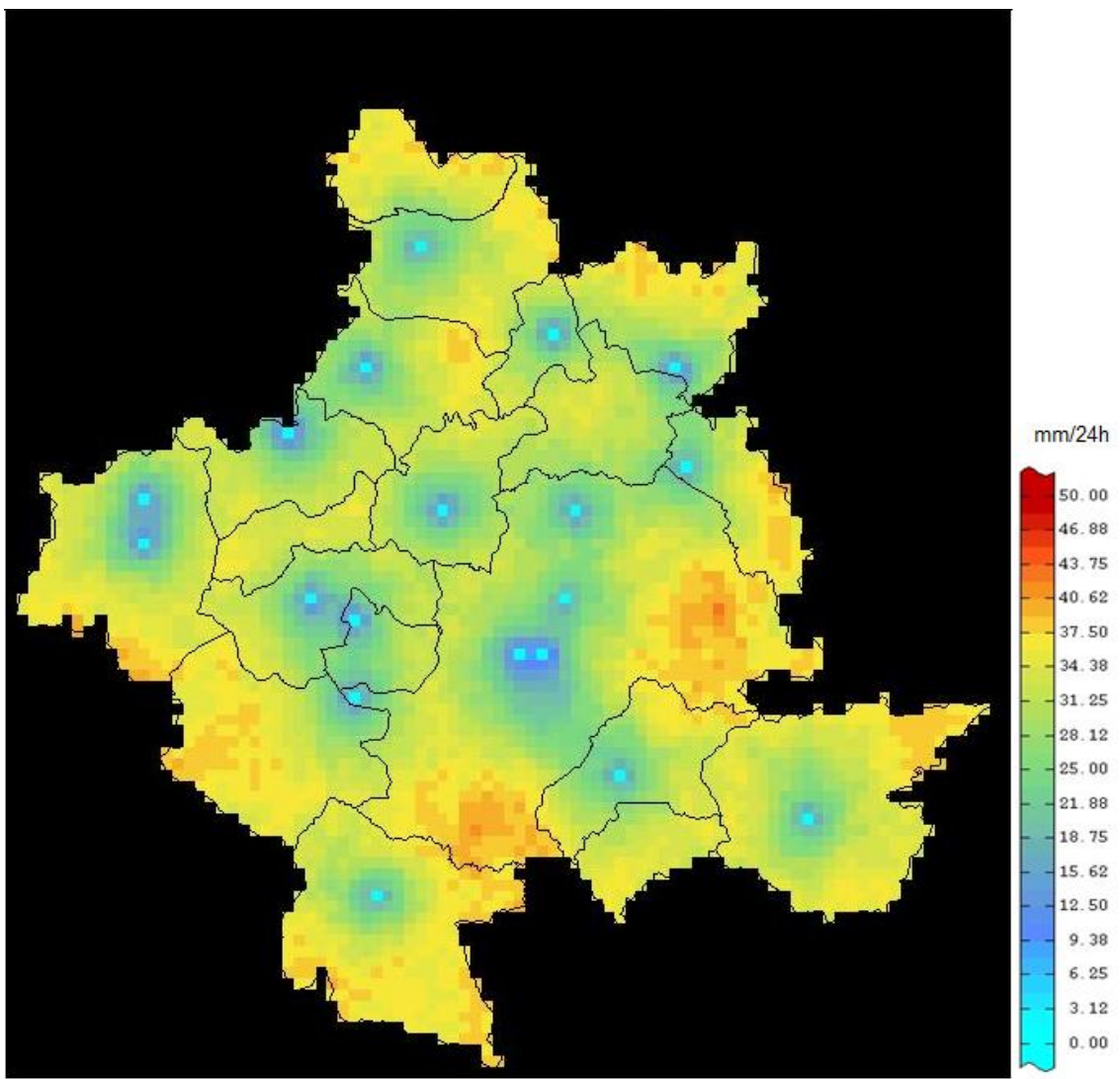

Figura 2. Mapa de desvio padrão da precipitação diária em Campinas em 24h.

Elaborado pelos autores

A RMC é servida por uma cobertura significativa de postos pluviométricos, contribuindo para a acurácia de informações sobre a dinâmica pluvial e, consequentemente, a maior parte da área apresenta grau moderado de incerteza, na faixa entre $34 \mathrm{~mm} / 24 \mathrm{~h}$ e $37 \mathrm{~mm} / 24 \mathrm{~h}$. Embora seja um montante moderado quando bem distribuído, pode provocar impactos significativos se ocorrer de forma concentrada.

Em Campinas são observados dois locais com alto grau de incerteza: nas regiões sul e sudeste, chegando a desvios de 50mm/24h. Considerando que a região sul apresenta conurbação com o município de Indaiatuba, é uma área a qual deve ser dada maior atenção pela confluência dos fatores de significativo 
XVII Simpósio Brasileiro

de Geografia Fisica Aplicada

I Congresso Nacional

de Geografia Física
OS DESAFIOS DA GEOGRAFIA FÍSICA NA FRONTEIRA DO CONHECIMENTO

Instituto de Geociências - Unicamp

Campinas - SP

28 de Junho à 02 de Julho de 2017

contingente populacional e menor certeza nas previsões. Alguns pontos desta área são ocupados por população com nível significativo de vulnerabilidade socioeconômica, o que se constituiu em outro fator de atenção.

Já a região sudeste é menos densamente urbanizada, ocupada, em sua maioria, por uma população de padrão de classe média a alta, com áreas de reservas ambientais e propriedades rurais de alto padrão, ou seja, caracteriza-se pela menor vulnerabilidade e maior resiliência.

As áreas com maior probabilidade de ocorrência de chuvas a partir de $80 \mathrm{~mm} / 24$, em condições atmosféricas análogas (Fig. 3), se concentram principalmente na parte central da RMC, com grande destaque para o município de Campinas. O local caracteriza-se por ser altamente urbanizado e com alta densidade populacional, onde as chuvas, mesmo não tão intensas, causam transtornos e perdas econômicas. Episódios de precipitação intensa não são raros na área, acarretando em impactos ainda mais graves.

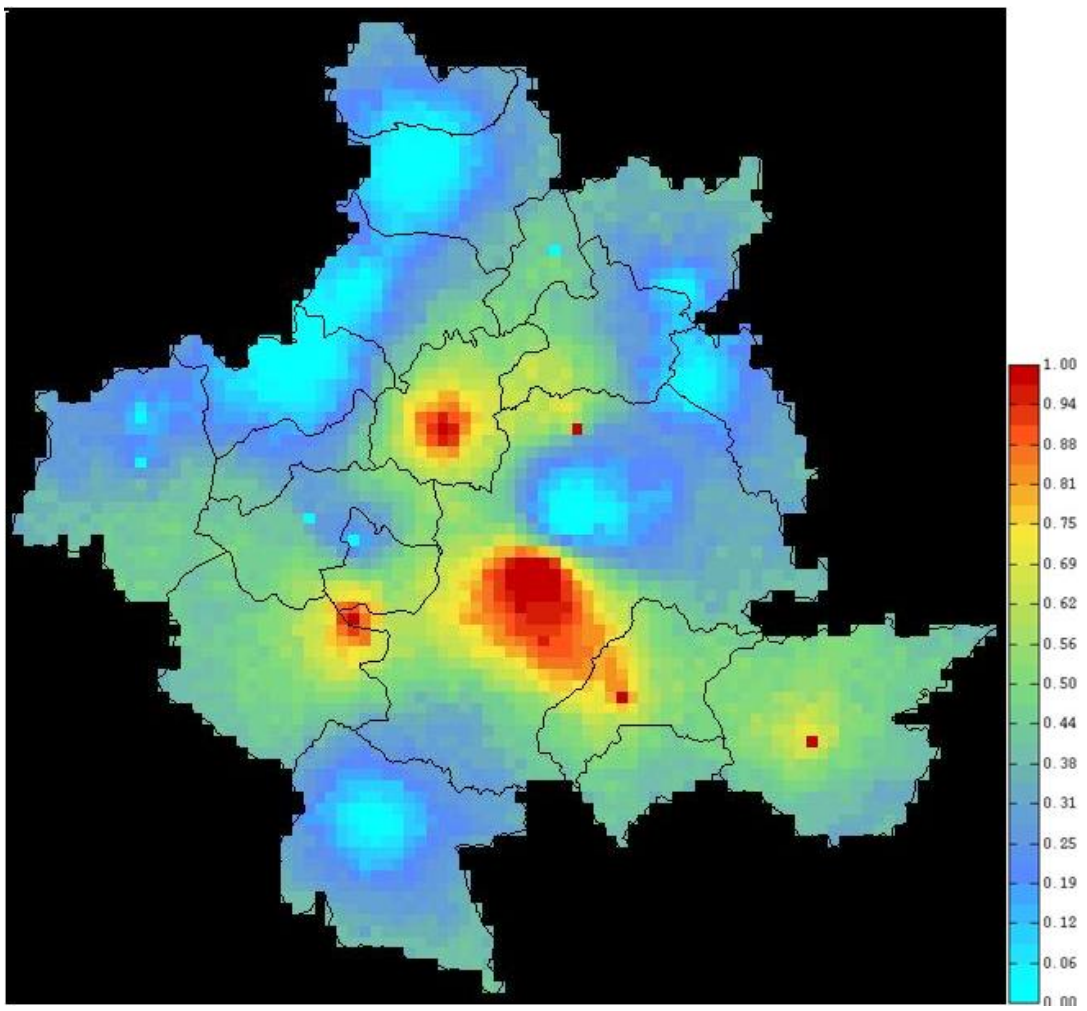

Figura 3. Regiões com probabilidade de ocorrência de chuvas a partir de $80 \mathrm{~mm} / 24 \mathrm{~h}$.

Elaborado pelos autores 




Destaca-se também o município de Paulínia, com alta probabilidade de chuvas intensas, que tem passado por grande expansão imobiliária nos últimos anos, com ocupação caracterizada por condomínios fechados de médio e alto padrão. Consequentemente, também aumenta o número de pessoas expostas ao risco.

A noroeste, destaca-se uma região com baixa probabilidade de chuvas intensas em condições semelhantes, composta pelos municípios de Arthur Nogueira, Cosmopolis e Americana. Além da baixa probabilidade, é igualmente uma área com baixa incerteza.

\section{Considerações Finais}

A técnica geoestatística de simulação estocástica foi eficiente como ferramentas para a análise da probabilidade de regiões da RMC serem atingidas por eventos pluviais intensos, bem como o grau de incerteza para a interpretação do mapa de limiares.

Os mapas de probabilidade fornecem tanto subsídio para tomadas de decisão frente à iminência de um evento pluvial de grande intensidade, apontando áreas mais suscetíveis à essas chuvas, quanto pode servir como um componente para elaboração de mapas de riscos, associado a outros elementos, tais como mapas hidrológicos. Salienta-se que neste estudo de caso foi considerada apenas a dimensão espacial para um dia, ou seja, os resultados aplicam-se apenas à casos em que as condições atmosféricas se configurem de forma análoga, contudo, esta metodologia quando aplicada às dimensões espacial e temporal (tratamento tridimensional) fornecerá um mapa climático de probabilidade de ocorrência de chuvas intensas.

Considera-se que a incorporação das incertezas em estudos às precipitações intensas pode ser uma ferramenta de grande auxílio no planejamento e gestão de riscos na escala municipal, contribuindo para a prevenção e minimização de prejuízos humanos e econômicos frente à essas ocorrências.

\section{Bibliografia}

ARAKI, R. Vulnerabilidade associada a precipitação e fatores antropogênicos na cidade do (São PauloBrazil): período de 1965 a 2001. 2007. (Mestrado em Geografia), Instituto de Geociências, Universidade de Campinas, Campinas/SP.

BABISH, G. Geostatistics without tears: a pratical guide to Surface interpolation, Geostatistics, variograms and Kriging. Saskatchewan/Canadá: Ecological Research. 2006. 116p.

CASTELLANO, M.S. Alagamentos em Campinas (SP) entre 1958 e 2007: tendências socioespaciais e ações governamentais. 2010 (Mestrado em Geografia), Instituto de Geociências, Universidade de Campinas, Campinas/SP. 




EM-DAT: The OFDA/CRED International Disaster Database (2010). Disponível em: < www.emdat.be - Université catholique de Louvain - Brussels - Belgium.

GOOVAERTS, P. Geostatistics for natural resources evaluation. New York; Oxford: Oxford University, 1997. 483p.

GUHA-SAPIR, D.; VOS, F.; BELOW, R; PONSERRE, S. Annual Disaster Statistical Review 2010: The Numbers and Trends. Brussels: CRED; 2011.

HELTON, J.C.; BURMASTER, D.E. Guest editorial: treatment of aleatory and epistemic uncertainty in performance assessments for complex systems. Reliability Engineering \& System Safety. Vol. 54, N. 2-3, pp. 91-262, 1996.

JOURNEL, A.G.; HUIJBREGTS, C.J. Mining geostatistics. London, New York: Academic Press. 1978, 600p.

LAVE, L.B.; APT, J. Planning for natural disasters in a stochastic world. Journal of Risk and uncertainty, v.33, p.117-130, 2006.

MORGAN, M.G.; HENRION, M. Uncertainty: A Guide to Dealing with Uncertainty in Quantitative Risk and Policy. Cambridge: Cambridge University Press. 1990. 330 p.

REMACRE, A.Z.; SACANI, S.S. Simulações Estocásticas. Notas de aula. 2005.

VICENTE, A. K. Eventos extremos de precipitação na Região Metropolitana de Campinas. 2005. Dissertação (Mestrado em Geografia), Instituto de Geociências, Universidade de Campinas, Campinas/SP.

ZANELLA, M. E.; SALES, M.C.L.; ABREU, N.J.A. Análise das precipitações diárias intensas e impactos gerados em Fortaleza, CE. GEOUSP (USP), v. 25, p. 53-68, 2009. 